\title{
STRUCTURAL VERSUS INDIVIDUAL EFFECTS ${ }^{1}$
}

\author{
ARNOLD S. TANNENBAUM AND JERALD G. BACHMAN
}

\begin{abstract}
Methods for separating the effects of group structure or composition from individual effects have been proposed by Blau and by Davis, Spaeth, and Huson. Both methods are useful, but they do not always hold individual or group characteristics strictly constant as intended, thereby making it possible to obtain spurious group or individual effects. Several modifications of these techniques are proposed, making use of more precise matching and correlational techniques. The advantages and limitations of each are discussed. Several additional problems are considered, including the effects of deviants, overlap of distributions among groups, selection processes, and the conceptual definition of structural or compositional effects.
\end{abstract}

One may define structural constructs as opposed to purely individual variables for purposes of group or organization theory. However, the frequent reliance, in empirical studies, on measures based on individual member responses often creates some operational ambiguity. Do the relationships observed when employing measures based on individual responses truly represent the effects of structural variables, or are they simply reflections of individuallevel relationships?

Blau has suggested one approach to this problem. He proposes an analytic technique which provides, in effect, an operational definition of structure. ${ }^{2}$ Davis, Spaeth, and Huson also provide an approach through the measurement of what they refer to as "compositional" effects. ${ }^{3}$ These approaches overlap in several essen-

\footnotetext{
${ }^{1}$ This article is written as part of a program of research on organizations under a grant from the Carnegie Corporation of New York to the Survey Research Center, Institute for Social Research, University of Michigan. We are indebted to the following friends and colleagues who kindly read an earlier draft and offered suggestions: David Bowers, Bruce Hill, Leslie Kish, Bernard Indik, John Kirscht, Philip Marcus, James Morgan, Frank Neff, Donald Pelz, Clagett Smith, and John Sonquist.

${ }^{2}$ Peter M. Blau, "Formal Organization: Dimensions of Analysis," American Journal of Sociology, LXIII (1957), 58-69, and his "Structural Effects," American Sociological Review, XXV (1960), 17893.
}

tial respects, and both represent significant contributions toward the solution of a difficult problem of sociological analysis. It is our intention to explore further the meaning of these methods, to consider some of their assumptions which appear to impose limitations on their applicability as presently formulated, and to suggest several means which may be helpful in reducing (if not overcoming) the effects of these limitations. Since Blau's approach is simpler in format, it will be easier to introduce the issues of the present paper primarily through reference to that approach. We shall then indicate their relevance to the method of Davis, Spaeth, and Huson.

Blau's strategy for determining structural effects may be summarized in three steps: ${ }^{4}$

1. An empirical measure, $Z$, is obtained that pertains to some characteristic of individual group members that has direct or indirect bearing upon the members' relations to each other (e.g., group identification, sociometric choices, initiation of interaction, rate of communication, or promotions).

2. The scores for measure $Z$, which

${ }^{3}$ James A. Davis, Joe L. Spaeth, and Carolyn Huson, "A Technique for Analyzing the Effects of Group Composition," American Sociological Review, XXVI (1961), 215-25.

* The following section closely paraphrases Blau, "Formal Organization ..., op. op. cit., p. 63. 
describes individuals, are combined into one index for each group, and this index no longer refers to any characteristic of individuals but to a characteristic of the group. The value of this index is presumed to vary across groups; we will define this variable as $Z_{\mathrm{gp}}$. Thus any individual may now be characterized in terms of his own score along variable $Z$ and his group's score along variable $Z_{\mathrm{gp}}{ }^{5}$

3. To isolate a structural effect, the relationship between the group attribute $\left(Z_{\mathrm{gp}}\right)$ and some dependent variable, $W$, is determined while the corresponding

TABLE $1 *$

PERFORMANCE SCORES BY RATE AND FREQUENCY OF DISCUSSION

(Hypothetical Example)

\begin{tabular}{|c|c|c|}
\hline \multirow{2}{*}{$\begin{array}{c}\text { Individuals Who Discuss } \\
\text { Their Problems }\end{array}$} & \multicolumn{2}{|c|}{$\begin{array}{l}\text { Groups Most of Whose } \\
\text { Members Discuss } \\
\text { Their Problems }\end{array}$} \\
\hline & Rarely & Often \\
\hline $\begin{array}{l}\text { Often } \ldots \ldots \ldots \ldots \ldots \\
\text { Rarely } \ldots \ldots \ldots \ldots \ldots\end{array}$ & $\begin{array}{l}0.65 \\
(1) \\
0.40 \\
(4)\end{array}$ & $\begin{array}{l}0.85 \\
(2) \\
0.70 \\
(3)\end{array}$ \\
\hline
\end{tabular}
p. 64 .

* Adapted from Blau, "Formal Organization ...," op. cit.,

characteristic of individuals $(Z)$ is held constant. The structural effect thus refers to the effect of $Z_{\mathrm{gp}}$ on $W$.

This method is illustrated by Blau through the hypothetical data of Table 1 in which five hundred persons are assumed to be arranged in fifty groups of about ten members each. We have numbered the cells

\footnotetext{
${ }^{5}$ Lazarsfeld and Menzel would define the $\boldsymbol{Z}_{\mathrm{gp}}$ variable in this usage as a "contextual property" of individuals, i.e., a property which stems from the individual's membership in a group (Paul F. Lazarsfeld and Herbert Menzel, "On the Relation between Individual and Collective Properties" in Amitai Etzioni [ed.], Complex Organizations [New York: Holt, Rinehart \& Winston, 1961], pp. 42240; see also Hannan C. Selvin and Warren O. Hagstrom, "The Empirical Classification of Formal Groups," American Sociological Review, XXVIII [1963], 399-411).
}

for convenience from 1 to 4 . Blau suggests that a structural effect is demonstrated by the differences in average performance scores between the two columns in Table 1. "This finding would show that, even when the effect of the individual's discussion rate of his problems on his performance is eliminated, just to be in a group where communication flows freely improves performance-other things being equal." 6 This statement, however, is based on an assumption which we must question.

The assumption of constancy within rows is asserted frequently by social researchers in relation to the type of analysis represented in Table 1. It can, however, lead to serious misinterpretations of data. It is important to recognize first of all that continuums underlie each of the axes in Table 1 , even though dichotomous categories are employed. Individuals (and groups) are not simply "often" or "rarely" communicators, but are likely to differ along a broad continuum of frequency of discussion. With this in mind, let us assume that all distributions within groups are normal (although almost any type of continuous distribution would lead to the same conclusion). The effects of this assumption can be seen in Figure 1. The points $Z_{1}-Z_{4}$ represent the average individual discussion scores of individuals in cells 1-4 of Table 1 . Several facts of importance are apparent from Figure 1:

1. In comparing individuals in cell 1 of Table 1 with those in cell 2, we are comparing individuals who have relatively low $\left(Z_{1}\right)$ discussion scores with those having higher $\left(Z^{2}\right)$ scores. We are not, in other words, holding the individual independent variable $(Z)$ constant, and cannot say that the difference between the two cells on the dependent variable represents the effects of social structure. The same problem applies to the comparison of the remaining two cells.

2. The failure to hold $Z$ strictly con-

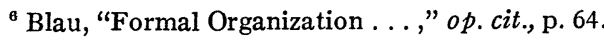


stant within rows has its counterpart in the failure to hold $Z_{\mathrm{gp}}$ constant within columns when more than two groups are being analyzed. The reader can see for himself how this unfortunate state of affairs develops by adding two normal frequency distributions, representing two additional groups, to the curves drawn in Figure 1. The pair of curves on the right would be labeled "High Discussion

$$
\begin{aligned}
Z_{1}= & \text { Average discussion score for indi- } \\
& \text { viduals high on frequency of dis- } \\
& \text { cussion in low-discussion group }
\end{aligned}
$$

$\mathrm{Z}_{2}=$ Average for high-discussion individuals in high group located. A more detailed and concrete illustration of this relationship and of the problems it creates can be seen from data which we have obtained employing Monte Carlo (random) techniques as follows: (a) A random sample of 150 individuals was drawn from a population which is normally distributed on individual variable $Z$. (b) This sample was randomly divided into fifty groups of three mem-

$$
\begin{aligned}
Z_{3} & =\text { Average for low individuals in } \\
& \text { high group } \\
Z_{4} & =\text { Average for low individuals in } \\
& \text { low group }
\end{aligned}
$$

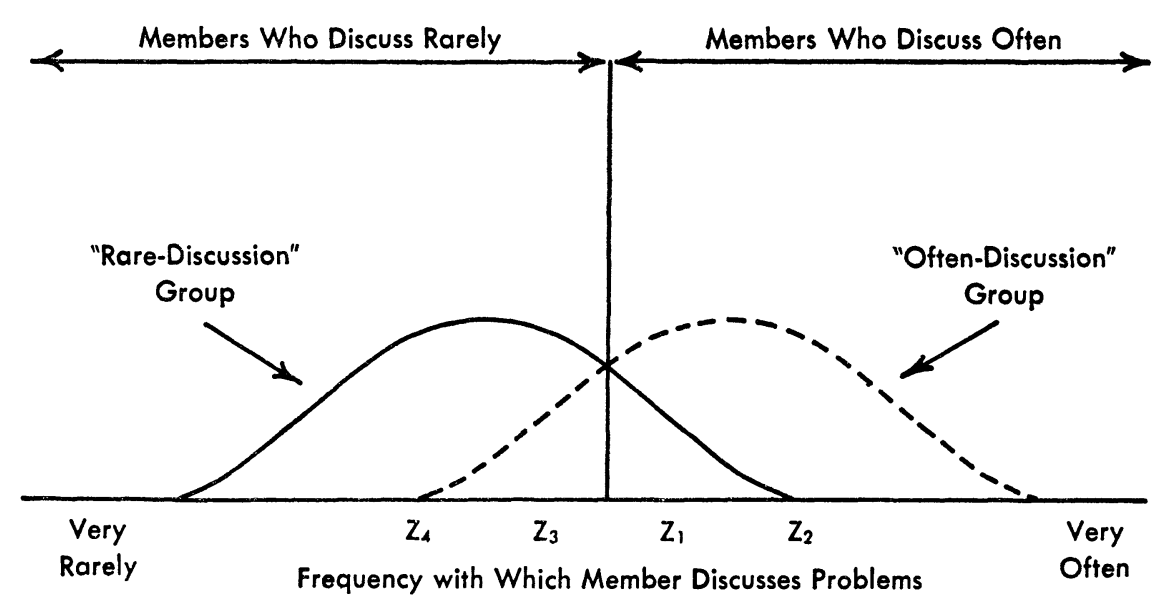

FIG. 1.-Hypothetical frequency distributions of members within two groups on a scale of frequency with which member discusses problems.

Groups." However, the one furthest to the right would contribute more members toward the computation of the mean in cell 2 than would the second group in that pair, while it would contribute fewer to the computation of the mean in cell 3 than would the second group. We would therefore be contaminating the individual-level (i.e., within-column) comparison with group effects.

Figure 1 implies a positive correlation between the $Z$ scores of individuals and the $Z_{\mathrm{gp}}$ scores assigned these individuals according to the groups in which they are bers each, and a $Z_{\mathrm{gp}}$ score (equal to the mean $Z$ for the three members) was derived for each group. Figure 2 presents the data obtained in this way. Each of the 150 "statistical individuals" is located in the matrix according to his own $Z$ score and the $Z_{\mathrm{gp}}$ score assigned to his group.

Let us define for these data a perfect linear relationship between the individual variable $Z$ and the dependent variable $W .^{7}$ Table 2 analyzes these data by the

\footnotetext{
${ }^{7}$ For the sake of clarity we have assumed a linear correlation of 1.00 between $Z$ and $W$. It is important to note, however, that the general observa-
} 
Blau method. According to this method the results would be interpreted as showing a strong individual-level effect coupled with a moderate (but quite definite) direct structural effect. However, we have defined dependent variable $W$ as being perfectly related to individual variable $Z$ and sibility of a genuine structural effect. The spurious structural effect indicated in Table 2 reflects the failure to hold the individual characteristic strictly constant within rows.

The processes underlying the problems noted above can be seen more clearly by

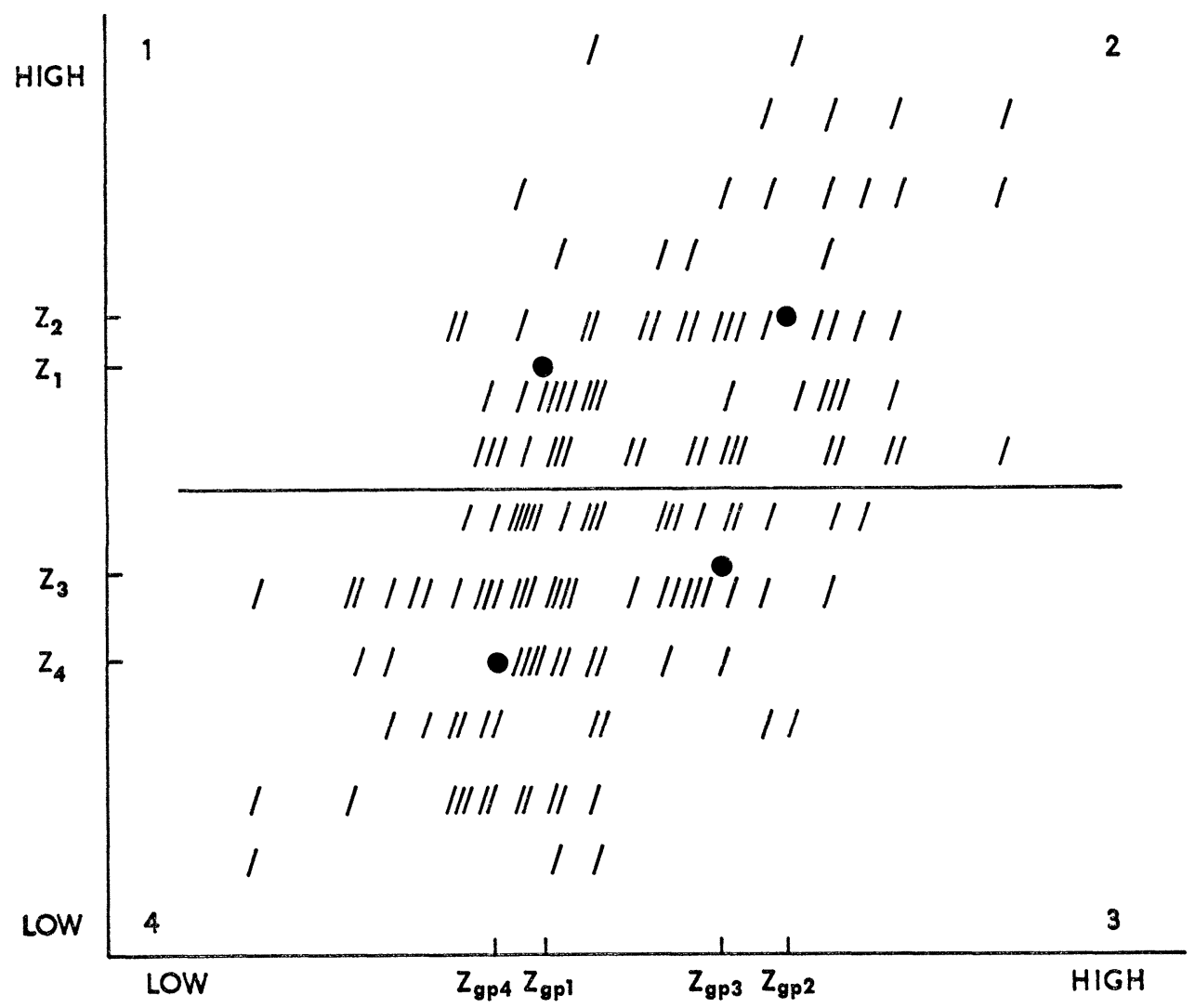

FIG. 2.-Scatter diagram showing $Z$ and $Z_{\mathrm{gp}}$ scores based on Monte Carlo data

have assigned members randomly to groups, thus effectively ruling out any pos-

tions which we will illustrate with these data apply equally well when there is any direct positive relationship between $Z$ and $W$. The use of a perfect correlation in our illustration simply serves to rule out random variation or "noise." Individual-level, curvilinear relationships between $Z$ and $W$ might lead spuriously to "contingency" or "inverse" type structural relationships described by Blau, depending upon the shape of the individual-level relationships. We are illustrating here a spurious "direct" type structural effect (see ibid.). returning to Figure 2. The intersecting lines in the diagram correspond to the dichotomies employed in deriving Table 2, and the four quadrants match the four cells in that table. The solid black circle in each cell indicates the mean $Z$ and $Z_{\mathrm{gp}}$ for those cases falling within the cell. It is apparent that the level of $Z$ for individuals in cell 1 is, on the average, lower than that for individuals in cell 2 . In other words, individual effects are not held strictly constant across the "high $Z$ " in- 
dividuals. And, of course, the same problem appears for the "low $Z$ " individuals in cells 3 and 4 .

The failure to hold group effects constant within columns can also be seen readily from this figure. "Low $Z_{\mathrm{gp}}$ " individuals in cell 4 come, on the average, from groups with lower $Z_{\mathrm{gp}}$ scores than do individuals in cell 1 ; and $Z_{\mathrm{gp}}$ scores are lower for "high $Z_{\mathrm{gp}}$ " individuals in cell 3 than for those in cell 2 .

The strategy used by Davis, Spaeth, and Huson is similar in several respects to that proposed by Blau. However, the former dichotomizes only on the $Z$ variable and not the $Z_{\mathrm{gp}}$. The groups are spread out along the horizontal axis according to their $Z_{\mathbf{g p}}$ scores. This eliminates the problem of contaminating within-column differences with group effects. However, the problem of eliminating individual effects in the intergroup comparisons remains. A limited solution to this problem, implicit in the Davis, Spaeth, and Huson method, is its restriction to individual characteristics that are dichotomous: "Within each population, individuals may be characterized by the presence or absence of a given independent attribute ( $A$ or $\bar{A})$." 8 To the extent that the individual variables involved are truly dichotomous, neither the Blau method nor that of Davis, Spaeth, and Huson need be concerned about the problem of controlling for individual effects. However, most variables of interest to social scientists (including some of those discussed by Davis et al.) are continuous, and the problem remains for these. In Figure 3 we apply the method of Davis, Spaeth, and Huson to our Monte Carlo data and see demonstrated (spuriously) a "Type IIIA" compositional effect: "a constant individual difference, along with a linear effect of group composition.",

${ }^{8}$ Davis et al., op. cit., p. 216 (italics as in original).

${ }^{\circ}$ Ibid., p. 220.
STRATEGIES FOR HOLDING CONSTANT INDIVIDUAL AND GROUP CHARACTERISTICS

The problems we have discussed stem from the assumptions that individual variables are held constant within rows and that group variables are held constant within columns. It is possible to reduce, if not to overcome, these problems through several modifications of the Blau or the Davis et al. methods. However, it is worth noting that the two problems may not be equally important in all situations. For example, a researcher who is interested

TABLE 2*

DEPENDENT VARIABLE $W$ AS RELATED TO INDIVIDUAL VARIABLE $Z$ AND GROUP VARIABLE $Z_{\mathbf{g p}}$

(Hypothetical Example-Blau Technique)

\begin{tabular}{|c|c|c|}
\hline \multirow{2}{*}{ INDIVIDUALS } & \multicolumn{2}{|c|}{ Groups } \\
\hline & Low $Z_{\mathrm{gp}}$ & High $Z_{\mathrm{gp}}$ \\
\hline $\begin{array}{l}\operatorname{High} Z \ldots \ldots \ldots \ldots \\
\text { Low } Z \ldots \ldots \ldots \ldots \ldots\end{array}$ & $\begin{array}{l}93.3 \\
(1) \\
49.5 \\
(4)\end{array}$ & $\begin{array}{c}100.4 \\
(2) \\
61.4 \\
(3)\end{array}$ \\
\hline
\end{tabular}
cell).

* Cell entries indicate mean $W$ (for all individuals in the

primarily in determining the presence of a structural effect may not be especially interested in whether a spurious individual-level effect appears as a result of his failure to hold group characteristics strictly constant. $\mathrm{He}$ will, on the other hand, be seriously concerned as to whether the structural effect he isolates is a spurious one caused by failure to hold individual characteristics constant. The techniques outlined below are not exhaustive, nor are they spelled out in fine detail. Our purpose is to open a number of avenues which may be useful in dealing with the problems raised above.

More precise matching of the individual variable.-The need for holding individual effects constant when comparing "high $Z_{\text {gp }}$ " 
and "low $Z_{\mathrm{gp}}$ " groups suggests that individuals be matched more closely on the individual independent variable $(Z)$. It should be noted that the fairly crude matching achieved when $Z$ is dichotomized represents a very great improvement over the situation which would exist if no attempt what- of categories to be used in any particular situation must be determined by the researcher.

Once the researcher has determined the number of categories into which to divide variable $Z$, he can proceed as in the Blau technique; he will, however, use

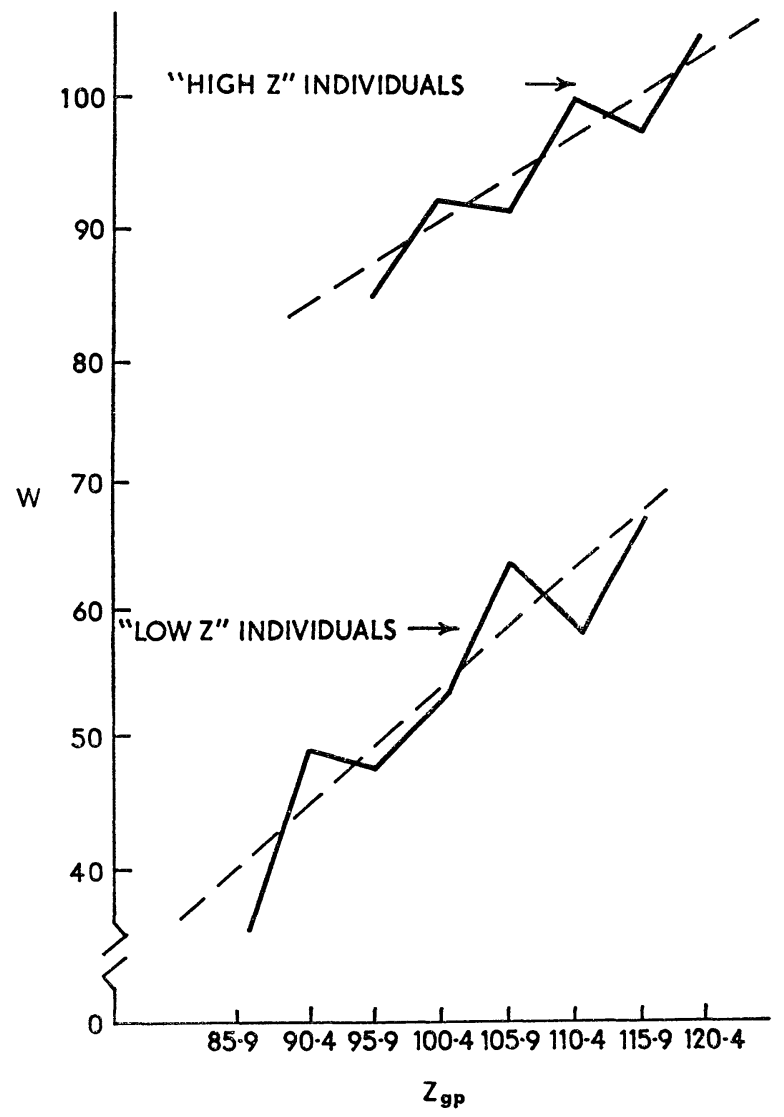

FIg. 3.-Dependent variable $W$ as related to individual variable $Z$ and group variable $Z_{\mathrm{gp}}$ (hypothetical example-Davis et al. technique).

ever were made to match individuals according to $Z$. However, as we have demonstrated, the dichotomy may not be sufficient. The larger the number of categories, of course, the greater the accuracy in matching; however, a "point of diminishing returns" is soon reached as the matching becomes more precise and as the number of cases falling within each category is reduced. The optimum number an $N \times 2$ rather than Blau's $2 \times 2$ table. Certain of the cells in such a table might be empty; these, as well as their counterparts in the opposite column, would have to be abandoned. The remaining cells will provide an estimate of structural effects with individual effects held (more or less) strictly constant. Returning to our random data, the application of this modification (using a $7 \times 2$ 
table rather than a $2 \times 2$ one) completely eliminates the spurious structural effect shown in Table 2. It may not, however, eliminate spurious individual effects.

The modified technique described above can be extended further so as to cover a broader range of scale points along the horizontal axis $\left(Z_{\mathrm{gp}}\right)$ in a manner suggested by Davis et al. This is preferable to the dichotomous analysis for several reasons. First, the dichotomy is usually inefficient statistically. Second, the use of a sufficient number of categories along the horizontal $\left(Z_{\mathrm{gp}}\right)$ dimension would hold group characteristics strictly constant and thus avoid the problem of spurious individual effects. Finally, the broader range of cases along the horizontal axis may lead to richer possibilities of analysis, increasing the likelihood of detecting the direct, inverse, and contingency effects discussed by Blau, or the various relationships in the typology outlined by Davis et al. Returning once again to our random data, the use of a $7 \times 8$ table rather than a $2 \times 8$ table would convert Figure 3 into a series of seven essentially horizontal lines, correctly indicating the presence of an individual, but not a structural, effect. However, the use of such a large number of cells drastically reduces the number of cases within each cell, so this variation will be appropriate only when the over-all number of cases is quite large.

Correlational methods.-Given a breakdown into $N$ levels of the individual variable $(Z)$ as described in the preceding section, it would be possible to determine the presence of structural effects by correlating $Z_{\mathrm{gp}}$ and $W$ at each of the $N$ levels of $Z$. This requires that each individual be assigned a $Z_{\mathrm{gp}}$ score according to the group in which he is located as well as his own individual $W$ score. In the case of our Monte Carlo data, we would have seven separate correlation coefficients (corresponding to the seven levels of individual variable $Z$ ). These correlations would not provide information about in- dividual-level effects. Such effects might be detected through the use of intragroup correlations, that is, by correlating $Z$ and $W$ separately within each group (thereby holding group effects constant).

Each of the above correlational procedures involves holding one variable constant while measuring the relationship between two others. If the particular data to be analyzed meet the necessary statistical requirements, the technique of partial correlation might achieve the same result. This could have the advantage of simplicity and precision. A structural effect could be measured in terms of the correlation between $Z_{\mathrm{gp}}$ and $W$ with $Z$ partialed out. An individual effect would be determined by the correlation of $Z$ and $W$ with $Z_{\mathrm{gp}}$ partialed out. ${ }^{10}$

A more thorough analysis of the dependent variable $W$ using $Z$ and $Z_{\mathrm{gp}}$ as the independent variables could be carried out through multiple-regression techniques. In such an approach, the change in $W$ expected with a unit change in $Z_{\mathrm{gp}}$ provides a measure of the structural effect, and the change in $W$ expected with a unit change in $Z$ provides a measure of the individual effect. It is very important in applying either this technique or that of partial correlation to remember the assumption of linearity upon which they are based. Unless the relationships between $Z, Z_{\mathrm{gp}}$, and $W$ are linear, the results of these analyses can be very misleading. However, it may sometimes be possible when the relationships are curvilinear to employ transformations, such as $Z^{2}$, log $W,\left(Z_{\mathrm{gp}}\right)^{2}$, and the like, to achieve the necessary linearity. ${ }^{11}$

${ }^{10}$ Thanks are due to Peter Blau for suggesting this possibility. Hubert M. Blalock, Jr., explains that "the partial correlation coefficient can be interpreted as a weighted average of the correlation coefficients that would have been obtained had the control variable been divided into very small intervals and separate correlations computed within each of these categories" (Social Statistics [New York: McGraw-Hill Book Co., 1960], p. 332).

${ }^{11}$ The multiple regression approach is somewhat related to L. A. Goodman's "Some Alternatives 
The correlational techniques described thus far are all concerned with predicting the dependent variable $(W)$ at the individual level. Another approach to detecting structural effects makes use of aggregate data such as those in the $N \times M$ table described in the preceding section. Given such a table, the correlations between $Z_{\mathrm{gp}}$ and mean $W$ can be determined at each of the $N$ levels of $Z$. In the case of the $7 \times 8$ table derived from our Monte Carlo data, we would have seven correlation coefficients (corresponding to the seven levels of individual variable $Z$ ). Each correlation would be based upon eight cells, with each cell referring to a certain level of $Z_{\mathrm{gp}}$ and the mean of the dependent variable $W$ for all individuals located in that cell.

Several cautions should be borne in mind in applying this method. First, while correlations based upon mean data can provide information about the overall presence or absence of a structural effect, they cannot be used to estimate how much of the variance in individuallevel $W$ can be related to $Z_{\mathrm{gp}}$. Second, correlations based upon aggregate data are not directly comparable to intragroup correlations since different $N$ 's and different groupings of the data are used; accordingly, their relative magnitudes do not indicate a relative strength of structural as compared to individual effects. Third, a correlation based upon a small number of data points (eight in our illustration) is subject to a great deal of variation due to chance, although this may be somewhat reduced when each of the points is based upon averages. Accordingly, any conclusion concerning the presence or absence of a structural effect should probably be based upon the overall pattern of correlations. ${ }^{12}$ On the posi-

to Ecological Correlation," American Journal of Sociology, LXIV (1959), 610-25 (see esp. pp. 623$25)$; and Dean Harper's Ph.D. dissertation ("Some New Applications of Dichotomous Algebra to Survey Analysis and Latent Structure Analysis" [Columbia University, 1961]). tive side, the use of aggregative instead of individual data may provide a more stable and accurate estimate of the true effect across groups, since each data point represents the observation of a number of individuals, thus eliminating a large portion of the random variance which occurs at the individual level. ${ }^{13}$

\section{SOME FURTHER CONSIDERATIONS}

A number of problems remain which apply to the original methods of Blau and of Davis $e t$ al. as well as to the modifications outlined in the preceding section.

The problem of overlap.-It can be seen through examination of Figure 1 and Table 1 that the $N$ 's in the four cells of the table are likely to be unequal, depending upon the extent to which the distributions of individual scores within the respective groups overlap. The $N$ 's in the four cells approach equality as the two distributions approach each other. But as this statistically desirable condition is approached, the data become meaningless as a basis for demonstrating structural effects; that is, structurally the groups are the same (on the independent variable) when the distributions coincide exactly. On the other hand, as the groups become more and more distinct, it is less and less possible to tell whether or not group effacts are present. The $N$ 's in cells 1 and 3 become zero when the two distributions do not overlap at all. This implies, in

${ }^{12} \mathrm{~A}$ weighted average correlation combining all of the correlations for each $Z$ level may sometimes be justified as a summary measure. In some cases it may be reasonable to derive a weighted average regression curve from the 7 (or $N$ ) curves, and a single, more stable correlation may be computed from this (see, e.g., A. S. Tannenbaum and C. G. Smith, "The Effects of Member Influence in an Organization: Phenomenology versus Organization Structure," Journal of Abnormal and Social Psychology, 1964 [in press]). While a single correlation obscures distinctions between the types of group compositional effects suggested by Davis et al. (op. cit., p. 219), it can indicate a general overriding trend of the data.

${ }^{13}$ Selvin and Hagstrom, op. cit. 
terms of the scatter diagram of Figure 2, a correlation between $Z$ and $Z_{\mathrm{gp}}$ approaching 1.00 . It is ironic that this situation, which seems conceptually most felicitous for the discovery of structural effects, precludes their detection by the methods under consideration.

Deviants.-A further qualification can be seen from Figure 1 and Table 1. Individuals in cells 1 and 3 are deviants within their respective groups (at least with respect to their scores on the independent variable), and their responses may be influenced by that fact alone. Thus, when we compare individuals in cell 2 with those in cell 1 we may be comparing "average" members in one group with "deviant" members in another. The same problem applies in the comparison of cells 3 and 4. The importance of this problem cannot be ascertained easily. One can hope that it is not a serious source of contamination in most cases, although we know that deviants are likely to be affected dfferently by group experiences than are average members. The researcher would probably do well to consider its possible effects in terms of the particular variables being analyzed.

Selection.-The manner in which members are selected into groups may influence the relationship between $Z_{\mathrm{gp}}$ and $W$ and may create in this way a spurious structural effect. For example, members of fraternities with high average intelligence $\left(Z_{\mathrm{gp}}\right)$ may have higher grade-point averages $(W)$ than members of low average intelligence fraternities, even when individual intelligence $(Z)$ is held strictly constant. This finding might be interpreted as indicating that being in a group of intelligent students creates better performance. Suppose, however, that certain fraternities maintain a policy of stressing high academic standing. Such a policy could lead to the selection of members directly on the basis of grades. Since intelligence and grades tend to be related, fraternities with such policies would be relatively high in average intelligence, thus producing the spurious structural relationship between average intelligence $\left(Z_{\mathrm{gp}}\right)$ and grade-point average $(W)$, while holding individual intelligence $(Z)$ constant.

It is probably worth keeping this problem in mind when interpreting group effects, since selection is a common phenomenon in social life. It is not unusual for individuals to join groups whose members are like themselves. Furthermore, even if selection into a group is random, selection out may be systematic, leaving a non-random selection behind. The various bases for selection may differ from case to case, and the corresponding interpretation of group effects would have to differ accordingly. Obviously, the problem can be completely eliminated in laboratory studies where groups are constructed by random procedures. Many field situations too would seem reasonably safe. The selection processes employed in creating formal work groups in industry, for example, are in many cases irrelevant to the particular variables under study, and these groups can be considered reasonably free of the problem. Certain informal and voluntary groups, however, may be more problematic, but this would depend again upon the variables under investigation.

Structural effects, operations versus concepts.-There is some conceptual haziness about variables which somehow are characterizations of both the organization and the individual. Research in group or organization functioning would do well to distinguish effects which are uniquely structural. While it may be easy enough to denote conceptually some variables that apply uniquely to structure and have no meaningful counterparts on the individual level, the fact that much social research must fall back upon measures based on individual responses creates a difficulty. While the concepts may be structural, the measures may be contaminated by individual effects. It is for this reason that the Blau method and that of Davis $e t a l$. are 
important approaches to the discovery of structural effects.

It is interesting to note, however, that Blau's original method, which is an operational approach to the definition of structural effects (and consequently structural characteristics), precludes from consideration, according to Blau, "those aspects of social structure which are not manifestations of frequency distributions, such as the form of government in the community."14 This type of variable, however, is obviously of great interest to the social researcher. Furthermore, the Blau method can be helpful in approaching this type of variable if it is employed not simply as a means of operationally defining structural variables and effects, but as a means of helping to ascertain whether the instrument chosen to measure a structural variable is in fact measuring such a characteristic.

We would like, therefore, to maintain the important distinction between a structural concept and a structural measure. While the concept, for example, may refer to aspects of the organization such as "chain of command," "flexibility," or "distribution of control," which are not manifestations of frequency distributions, the measures may very well be based on distributions, that is, on the responses of individual members. ${ }^{15}$ Measures of these

\footnotetext{
${ }^{14}$ Blau, "Structural Effects," op. cit., p. 192.

${ }^{15}$ See, e.g., Ellis L. Scott, who is concerned with the causes of error in the perception of the "chain of command" (Leadership and Perceptions of Organizations [Research Monograph No. 82 (Ohio State University, Columbus: Bureau of Business Research, Ohio State University, 1956)]). Basil Georgopolous and Arnold S. Tannenbaum measure organizational flexibility by averaging responses of organization members to questions designed to provide estimates of this variable ("A Study of Organizational Effectiveness," American Sociological Review, XXII [October, 1957], 534-40). Martin Patchen is concerned with the validity of measures, based on member responses, of distribution of control in organizations ("Alternative Questionnaire Approaches to the Measurement of Influences in Organizations," American Journal of Sociology, LXIX [July, 1963], 41-52).
}

structural concepts would be subsumed under Kendall and Lazersfeld's unit datum of Type V where "the unit item characterized the group only" and where "no information is introduced about a single individual." 16 We add simply that, while no information may be introduced about a single individual, information may be introduced $b y$ individuals. It is for this reason that the Blau method and that of Davis et al. can prove helpful.

Structural variables should be chosen first on the basis of their theoretical meaningfulness. Measurement is a second step, and tests of relationships between these variables and others are a third. Measurement of a pure structural effect in this sense might then be gauged by the occurrence of a difference between groups according to one of the above methods and a zero difference within groups. This is, with some modification, the Type II effect described by Davis et al. Conceptually, we would attempt to approach in this way the effect of a structural variable which has no meaningful counterpart on the individual level-although all of our measures are obtained at that level.

\section{SUMMARY AND CONCLUSIONS}

Since measures of group and organization variables are often based on responses of individuals, it is sometimes difficult to know whether the effects observed are due to structure or due simply to individual characteristics. Blau has suggested a useful approach to this difficulty, but one that appears to contain two problems: (1) it fails to hold individual characteristics strictly constant and thereby makes it possible to obtain spurious structural effects; (2) it fails to hold group characteristics

\footnotetext{
${ }^{16}$ Patricia L. Kendall and Paul F. Lazarsfeld, "Problems of Survey Analysis," in Robert K. Merton and Paul F. Lazarsfeld (eds.), Continuities in Social Research (Glencoe, Ill.: Free Press, 1950), pp. 133-96. See also Selvin and Hagstrom's discussion (op. cit.) of aggregative and integral properties of groups and their distinction between members as respondents and as informants.
} 
strictly constant, making possible the occurrence of spurious individual-level effects. A technique similar in some respects to Blau's has been developed by Davis et $a l$; this method is susceptible only to problem (1).

We have proposed several modifications of the Blau and the Davis et al. methods, making use of more precise matching and correlational techniques. Each of these modified methods involves certain advantages and limitations, and the researcher may want to employ them in combination or modify them further to suit his particular purposes.

Several additional problems have been considered including the effect of deviants, overlap of distributions among groups, and selection. We were also concerned about the purpose of the original methods discussed here, namely, defining structural or compositional effects (and, by implication, structural variables) operationally. In the authors' opinion this is not an adequate substitute for the conceptual definition of structural variables; conceptualization should come first. The application of the above techniques could then serve the very useful function of determining whether or not the operations employed can be justified as measures of structural characteristics and effects.

Survey Research Center UNIVERSITY OF MICHIGAN 